\title{
Estimación y modelización de arrastres de orgánico en acuoso (O/A) en pruebas de laboratorio de planta de extracción por solventes (SX) de cobre ${ }^{(\bullet)}$
}

\author{
O. Benavente*, M.C. Hernández* y F. Sagredo*
}

\begin{abstract}
Resumen Las pérdidas de orgánico a través de los arrastres $(\mathrm{O} / \mathrm{A})$ en la extracción por solventes implica una merma económica: por tal razón surge el interés de estimar el efecto que tienen tres oximas diferentes bajo distintas condiciones operacionales, además, modelizarlas en función de variables físico-químicas, de manera que se puedan predecir estas pérdidas. Las pruebas se realizan en un reactor Anetich en continuidad acuosa usando diluyente Orfom SX 12, con concentraciones de extractante de $10 \%, 15 \%$ y $20 \%$. Para la confección del modelo de medias se aplica un diseño factorial $2^{\mathrm{k}}$ a las pruebas. Los resultados indican que la cetoxima presenta en promedio un $40 \%$ y $20 \%$ menos arrastre que la aldoxima y la mezcla respectivamente. Además el modelo está dado por la ecuación Arrastre O/A = C - B* Temperatura - E* pH + F* Agitación; donde $\mathrm{C}, \mathrm{B}, \mathrm{E}$ y $\mathrm{F}$ son constantes específicas de cada reactivo.
\end{abstract}

Palabras Clave $\quad$ Arrastre; Extracción por solventes; Modelo; Oxima; Orgánico.

\section{Estimation and modeling of organic entrainment in aqueous solution (O/A) in laboratory tests of copper solvent extraction (SX) plant}

\begin{abstract}
The losses of organic through entrainment $(\mathrm{O} / \mathrm{A})$, in the solvent extraction process, implies economic waste; for such reason we became interested in estimating the effect with three different oximes under different operational conditions, in addition, to model them in terms of physico-chemical variables, so that these losses can be predicted. The tests are made in Anetich reactor in aqueous continuity using Orfom SX 12 diluent, with the following concentrations of extracting agent $10 \%, 15 \%$ and $20 \%$. For the preparation of the measures model a factorial design is applied $2^{\mathrm{k}}$ to the tests. The results indicate that ketoxime displays a minor entrainment of $\mathrm{O} / \mathrm{A}$ in average $40 \%$ and $20 \%$ less in comparison with aldoxime and the mixture respectively. The model is given by the equation: Entrainment of $\mathrm{O} / \mathrm{A}=\mathrm{C}-\mathrm{B} *$ Temperature $-\mathrm{E} * \mathrm{pH}+\mathrm{F} *$ Agitation; where $\mathrm{C}, \mathrm{B}, \mathrm{E}$ and $\mathrm{F}$ are specific constants of each reagent.
\end{abstract}

Keywords

Entrainment; Solvent extraction; Model; Oxime; Organic.

\section{INTRODUCCIÓN}

Las plantas hidrometalúrgicas cuentan con una serie de equipos internos y anexos a SX, los cuales permiten atenuar las pérdidas de reactivo, disminuyendo así los efectos perjudiciales en otras etapas producidas por el orgánico arrastrado; entre estos equipos se pueden mencionar los "picked fence", distribuidores de flujo, pos decantadores, cintas oleo-filicas en piscinas de refino y estanques de electrolito rico, filtros Chuquicamata y a presión (Spintek y Delkor), coalescedores, columnas de flotación Magma, celdas de sacrificio en electro-obtención, etc. Sin embargo, todas estas medidas de mitigación disminuyen las pérdidas de reactivo pero no eliminan totalmente los problemas generados por los arrastres.

El $96 \%$ de las pérdidas por arrastres $\mathrm{O} / \mathrm{A}$ se generan en la etapa de extracción y el $4 \%$ en la de re-extracción ${ }^{[1]}$ y se encuentran directamente relacionadas con la distribución de tamaño de gotas en el mezclador. Dicha distribución del tamaño de gotas, es consecuencia de dos procesos: la rotura de gota debido a la velocidad de disipación de la energía en la fase continua y la coalescencia entre las gotas. La velocidad de los procesos de rotura y la coalescencia de la gota están gobernadas por la tensión interfacial de ambas fases ${ }^{[2]}$.

Las variables de temperatura, agitación y $\mathrm{pH}$ tienen efecto en la coalescencia de las gotas. A medida

\footnotetext{
(•) Trabajo recibido el día 1 de marzo de 2012 y aceptado en su forma final el día 3 de agosto de 2012.

Departamento de Metalurgia y Minas, Universidad Católica del Norte, Chile.
} 
que aumenta la velocidad de agitación se tiende a reducir la coalescencia de las gotas debido a que aumenta el tiempo de separación de fases ${ }^{[3 \text { y } 4]}$. La densidad y viscosidad también afectan a la coalescencia de las gotas: estudios recientes indican que es más probable que se formen gotas secundarias que micro-gotas con aceites de baja viscosidad ${ }^{[5]}$.

A alto $\mathrm{pH}$ la coalescencia se ve obstaculizada debido al efecto electroquímico de doble capa alrededor de las gotas ${ }^{[6]}$. Al aumentar la temperatura disminuye la viscosidad de la fase dispersa y la tensión interfacial del sistema ${ }^{[3]}$.

Los arrastres en SX son consecuencia de las interacciones entre variables; es por ello que se utilizan técnicas de análisis de multi-variable, tal como es el diseño y análisis factorial que permite modelizar los resultados obtenidos, a través de un modelo de medias. El modelo de medias en su modo general establece que $Y_{\mathrm{ijk}}=\mu_{\mathrm{ij}}+\mathrm{e}_{\mathrm{ijk}}$, siendo la media de la celda ij-ésima; $Y_{\mathrm{ijk}}=\mu+\tau_{\mathrm{i}}+\beta_{\mathrm{j}}+(\tau \beta)_{\mathrm{ij}}+\varepsilon_{\mathrm{ijk}}$, donde $\mu$ es el efecto promedio global, $\tau_{i}$ es el efecto del nivel i-ésimo del factor $A$ de los renglones, $\beta_{\mathrm{j}}$ es el efecto del nivel $\mathrm{j}$-ésimo del factor $\mathrm{B}$ de las columnas, $(\tau \beta)_{\mathrm{ij}}$ es el efecto de la interacción entre $\tau_{\mathrm{i}}$ y $\beta_{\mathrm{j}}$ y $\varepsilon_{\mathrm{ijk}}$ es un componente de error aleatorio.
El presente trabajo de investigación tiene como objetivo estimar y modelizar los arrastres $\mathrm{O} / \mathrm{A}$ a escala de laboratorio bajo diferentes condiciones operacionales utilizando tres oximas distintas.

\section{METODOLOGIA EXPERIMENTAL}

Para la evaluación de los arrastres, se emplea un reactor Anetich con control de temperatura. Para la toma de muestra se implementó una técnica la cual consiste en ingresar un dispositivo por la zona superior del reactor. La cuantificación de los arrastres se realiza por absorción atómica. Se desarrolla un diseño factorial que permite estimar los arrastres en distintas condiciones operacionales y modelizarlos a través de un modelo de medias.

La tabla I muestra los niveles máximos y mínimos así como los parámetros sometidos al diseño factorial.

Los niveles mínimos y máximos del $\mathrm{pH}$ varían entre 1,6 y 2,0; la agitación entre 500 y 700 rpm y la temperatura entre los 10 y $20^{\circ} \mathrm{C}$.

La tabla II muestra los parámetros operacionales de las pruebas.

Tabla I. Factores del diseño factorial máximos y mínimos

Table I. Maximum and minimum factors of the factorial design $2^{k}$

\begin{tabular}{lcc}
\hline \multicolumn{1}{c}{ Variable } & Nivel mínimo & Nivel máximo \\
\hline Agitación & -1 & 1 \\
Temperatura & -1 & 1 \\
$\mathrm{pH}$ & -1 & 1 \\
\hline
\end{tabular}

Tabla II. Parámetros operacionales para las pruebas

Table II. Operational parameters for the experimental test

\section{Parámetros}

\begin{tabular}{lc}
\hline Concentración Cu PLS $(\mathrm{g} / \mathrm{L})$ & 6 \\
Diluyente & Orfom SX 12 \\
Agitación (rpm) & 500 y 700 \\
$\mathrm{pH}$ & 1,6 a 2,0 \\
Continuidad & Acuosa \\
O/A & $1: 1$ \\
Temperatura ${ }^{\circ} \mathrm{C}$ & 10 y 20 \\
Volumen de orgánico $(\mathrm{mL})$ & 400 \\
Volumen de acuoso $(\mathrm{mL})$ & 400 \\
\hline
\end{tabular}


Para la evaluación y estimación de arrastre se utilizan tres extractantes comerciales basados en aldoxima, mezcla de aldoxima/cetoxima y cetoxima, denominados como A, B y C, preparados con una concentración de 10, 15 y $20 \% \mathrm{~V} / \mathrm{V}$ en solvente Orfom SX 12. Las pruebas consideran al orgánico como fase dispersa ya que es la condición más desfavorable desde el punto de vista operacional para minimizar la generación de arrastres $\mathrm{O} / \mathrm{A}$.

Los resultados del diseño factorial son analizados con el software estadístico Minitab v.15 el cual permite desarrollar el modelo de medias.

\section{RESULTADOS Y DISCUSIÓN}

\subsection{Caracterización de la fase orgánica}

La tensión interfacial es determinada utilizando el anillo de Dunouy, la densidad usando un densímetro y la viscosidad se mide con el viscosímetro de caída de bola Gilmont. La tabla III muestra los valores obtenidos en la fase orgánica.

La densidad y la viscosidad son mayores para la oxima A (Tabla III); esto se debe a la presencia del modificador. Además, se observa que al aumentar la temperatura ambas propiedades disminuyen.

La tabla IV muestra los resultados de la tensión interfacial. Se observa una tendencia a disminuir la tensión a medida que aumenta la concentración de extractante y la temperatura, a consecuencia de la disminución de las fuerzas inter-iónicas entre las gotas de ambas fases. Resultados similares se obtienen en trabajos de investigación de sistemas líquidos - líquidos ${ }^{[7-9]}$. Además, la tensión interfacial disminuye a medida que aumenta el $\mathrm{pH}$ y la concentración de extractante. Tal situación se debe a que en la superficie de las gotas orgánicas los iones negativos de hidróxidos son especial y espontáneamente absorbi$\operatorname{dos}^{[10]}$.

\subsection{Efecto de la agitación en los arrastres O/A}

A medida que aumenta la agitación, se incrementan los arrastres O/A en todas las concentraciones de extractante (Fig.1). Esta situación se debe a que al aumentar la agitación se favorece la generación de micro-gotas que no logran coalescer y quedan atrapadas en una fase distinta. Esta hipótesis es compartida con diferentes trabajos de investigación ${ }^{[3}$ y 4$]$

Tabla III. Densidad y viscosidad de las soluciones orgánicas

Table III. Density and viscosity of solution

\begin{tabular}{lccc}
\hline & Temperatura, $^{\circ} \mathbf{C}$ & Densidad, $\mathbf{g} / \mathbf{m L}$ & Viscosidad, $\mathbf{C p}$ \\
\hline $10 \%$ A & 10 & 0,816 & 2,81 \\
$10 \%$ B & 10 & 0,813 & 2,558 \\
$10 \%$ C & 10 & 0,811 & 2,415 \\
$15 \%$ A & 10 & 0,821 & 3,075 \\
$15 \%$ B & 10 & 0,816 & 2,882 \\
$15 \%$ C & 10 & 0,816 & 2,773 \\
$20 \%$ A & 10 & 0,827 & 3,453 \\
$20 \%$ B & 10 & 0,824 & 3,274 \\
$20 \%$ C & 10 & 0,821 & 3,24 \\
$10 \%$ A & 20 & 0,808 & 2,705 \\
$10 \%$ B & 20 & 0,793 & 2,349 \\
$10 \%$ C & 20 & 0,79 & 2,313 \\
$15 \%$ A & 20 & 0,817 & 2,99 \\
$15 \%$ B & 20 & 0,815 & 2,558 \\
$15 \%$ C & 20 & 0,815 & 2,378 \\
$20 \%$ A & 20 & 0,823 & 3,239 \\
$20 \%$ B & 20 & 0,821 & 3,096 \\
$20 \%$ C & 20 & 0,82 & 2,988 \\
\hline
\end{tabular}


Tabla IV. Tensión interfacial

Table IV. Interfacial tension

\begin{tabular}{|c|c|c|c|c|c|c|c|c|c|}
\hline \multirow{2}{*}{ Fase acuosa } & \multicolumn{8}{|c|}{ Fase orgánica (dina / cm) } & \multirow[b]{2}{*}{$20 \% \mathrm{C}$} \\
\hline & $10 \%$ & $10 \% B$ & $10 \% C$ & $15 \% A$ & $15 \% B$ & $15 \% C$ & $20 \% \mathrm{~A}$ & $20 \% \mathrm{~B}$ & \\
\hline $\begin{array}{l}6 \mathrm{~g} / \mathrm{L} \mathrm{Cu}-\mathrm{pH} 1,6- \\
10^{\circ} \mathrm{C}\end{array}$ & 15,5 & 16,1 & 19,3 & 15,2 & 15,7 & 17,5 & 15,7 & 15,0 & 15,0 \\
\hline $\begin{array}{l}6 \mathrm{~g} / \mathrm{L} \mathrm{Cu}-\mathrm{pH} 1,6- \\
20^{\circ} \mathrm{C}\end{array}$ & 15,0 & 14,3 & 18,5 & 14,6 & 14,7 & 16,1 & 15,4 & 14,8 & 14,8 \\
\hline $\begin{array}{l}6 \mathrm{~g} / \mathrm{L} \mathrm{Cu}-\mathrm{pH} 2,0- \\
10^{\circ} \mathrm{C}\end{array}$ & 15,1 & 15,5 & 18,4 & 14,9 & 15,1 & 17,4 & 14,9 & 14,7 & 14,7 \\
\hline $\begin{array}{l}6 \mathrm{~g} / \mathrm{L} \mathrm{Cu}-\mathrm{pH} 2,0- \\
20^{\circ} \mathrm{C}\end{array}$ & 14,8 & 14,2 & 17,0 & 14,1 & 14,3 & 15,8 & 15,1 & 14,6 & 14,6 \\
\hline
\end{tabular}

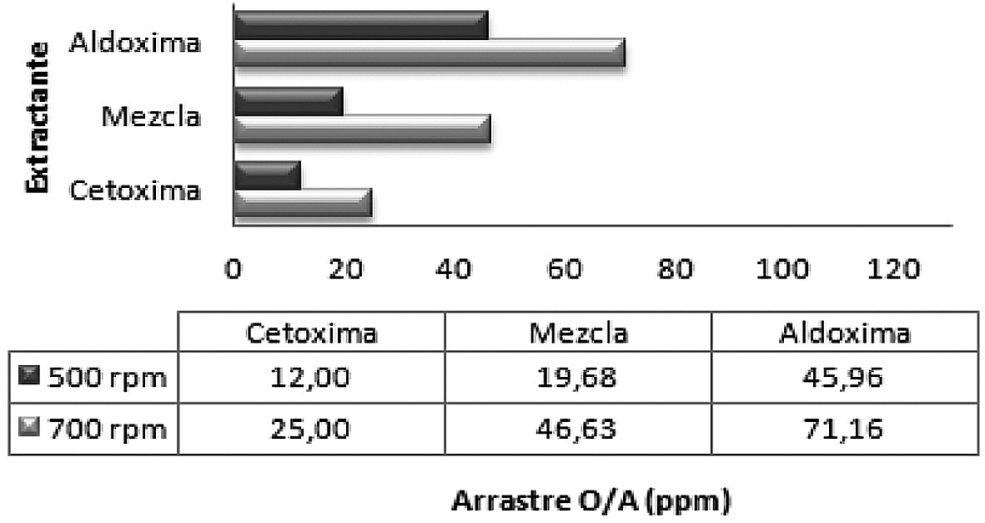

Figura 1. Efecto de la agitación en los arrastres O/A de A, B y C al $15 \%$ de extractante y $20^{\circ} \mathrm{C}$.

Figure1. Effect of the agitation in entrainment $O / A$ by $A, B$ and C with $15 \%$ of extractant and $20^{\circ} \mathrm{C}$.

La oxima $\mathrm{A}$, presenta el nivel más alto de arrastre O/A, seguida por la oxima B y la C. Esta situación se explica ya que presenta una mayor viscosidad, respecto a las otras oximas evaluadas. Por tal razón es más probable que se formen micro-gotas que dificultan su coalescencia ${ }^{[5]}$.

\subsection{Efecto de la temperatura en los arrastres O/A}

En la figura 2 se aprecia que a medida que aumenta la temperatura, se incrementan los arrastres O/A, a cualquier concentración de extractante.
Esta situación se debe a que al aumentar la temperatura disminuye la tensión interfacial. Respecto a la distribución de tamaño de las gotas, éstas tienden a ser más pequeñas al incrementar la temperatura. Resultados similares se han obtenido en trabajos de investigación en sistemas líquido - líquido ${ }^{[3,7-9]}$.

\subsection{Efecto del pH en los arrastres O/A}

En la figura 3 se aprecia que a medida que aumenta el $\mathrm{pH}$, se incrementan los arrastres O/A; esta situación se asocia a la disminución de la tensión interfacial. 


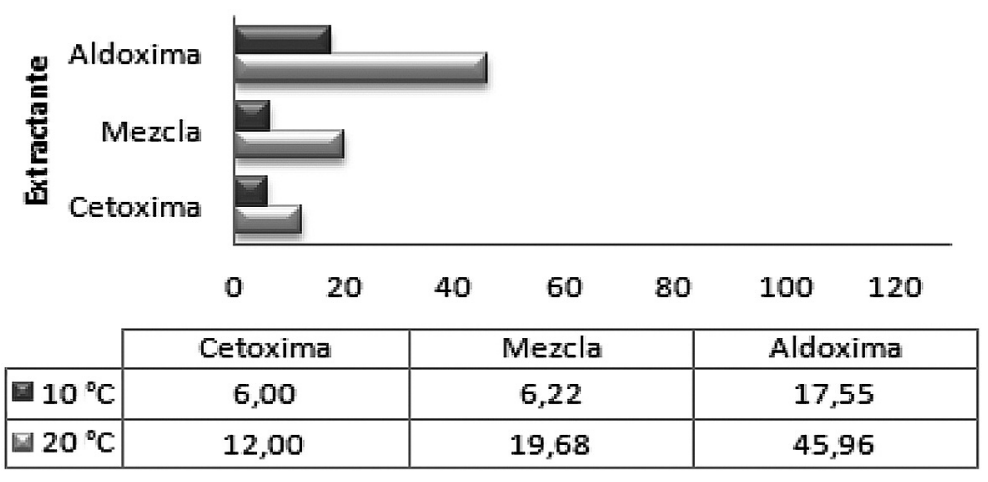

Arrastre O/A (ppm)

Figura 2. Efecto de la temperatura en los arrastres O/A de A, B y $\mathrm{C}$ al $15 \%$ de extractante y $500 \mathrm{rpm}$.

Figure 2. Effect of the temperature in entrainment O/A by $A$, $B$ and $C$ with $15 \%$ of extractant and $500 \mathrm{rpm}$ of agitation.

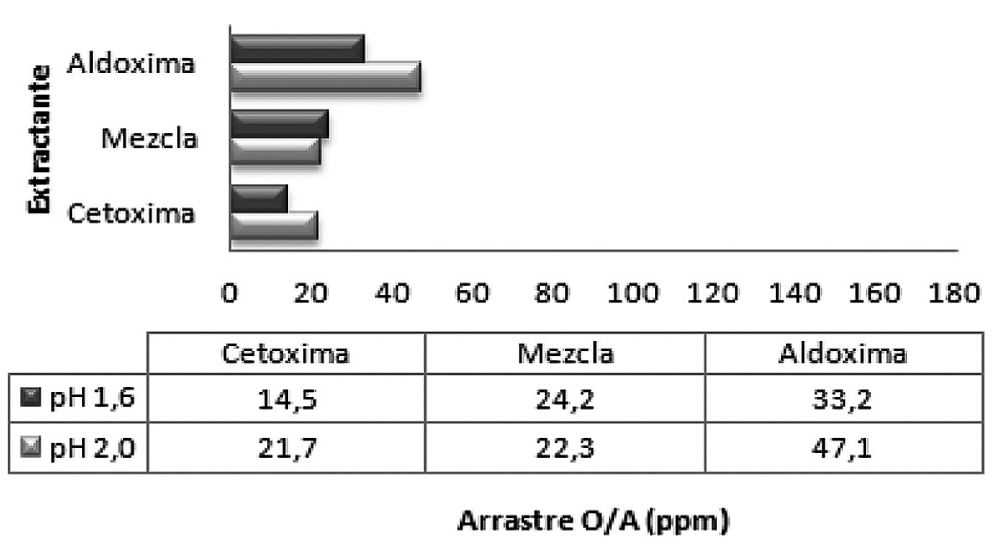

Figura 3. Efecto del $\mathrm{pH}$ en los arrastres $\mathrm{O} / \mathrm{A}$ de $\mathrm{A}, \mathrm{B}$ y $\mathrm{C}$ al $15 \%$ de extractante y $25^{\circ} \mathrm{C}$.

Figure 3. Effect of the $\mathrm{pH}$ in entrainment $\mathrm{O} / \mathrm{A}$ by $A, B$ and $C$ with $15 \%$ of extractant and $25^{\circ} \mathrm{C}$.

El aumento de arrastre O/A por efecto del $\mathrm{pH}$ se contrapone a los resultados obtenidos por otros autores $^{[3]}$. Sin embargo, autores que estudiaron el efecto del $\mathrm{pH}$ en la coalescencia de las gotas en fase acuosa usando benceno y tetracloruro de carbono, lograron establecer que el $\mathrm{pH}$ inhibe la coalescen$\mathrm{cia}^{[10]}$.

\subsection{Modelización de los resultados}

La modelización de los arrastres O/A se realiza utilizando un modelo de medias que se obtiene del diseño factorial, el cual en su forma general presenta la siguiente ecuación (Ec. (1)):

$$
Y_{i j k}=\mu+\tau_{i}+\beta_{j}+(\tau ß)_{i j}+\varepsilon_{i j k}
$$

El planteamiento de hipótesis del modelo establece (Ecs. (2), (3) y (4)):

$$
\text { Hipótesis nula } \mathrm{H}_{\mathrm{o}}=\tau_{1}=\tau_{2}=\tau_{\mathrm{n}}
$$

Hipótesis alternativa $\mathrm{H}_{1} \neq \tau_{1} \neq \tau_{2} \neq \tau_{\mathrm{n}}$

$$
\text { Hipótesis nula } H_{0}=\beta_{1}=\beta_{2}=\beta_{n}
$$

Los supuestos del modelo consideran que los errores y/o residuos deben ser:

- Independientes

- Idénticamente distribuidos normalmente 
- Media cero y varianza constante

El criterio de rechazo de la hipótesis nula se basa en el p-valor, como se observa en la ecuación $\mathrm{P}$-valor $\leq \alpha$. No se rechaza la hipótesis nula donde $\alpha$ es 0,05 ; representa un $95 \%$ de confianza en los resultados. En todos los modelos se logra un p-valor mayor a 0,05 .

Se obtienen las siguientes ecuaciones del modelo de medias para los reactivos $\mathrm{A}, \mathrm{B}$ y $\mathrm{C}$, respectivamente (Ecs. (5), (6) y (7)):

\section{A: Arrastre O/A= 55,8 - 19,7 Temperatura -}

$$
\text { - 11,4 pH + 17,1 Agitación }
$$

\section{B: Arrastre $\mathrm{O} / \mathrm{A}=28,8-10,2$ Temperatura - - 3,35 pH + 7,14 Agitación}

\section{C: Arrastre O/A = 9,69 - 2,64 Temperatura -}

$$
-1,73 \mathrm{pH}+3,55 \text { Agitación }
$$

De las ecuaciones se obtiene que cualquiera que fuesen la temperatura, $\mathrm{pH}$ y agitación, la oxima $\mathrm{A}$ presentará mayor arrastre. La comprobación del modelo se realiza utilizando los errores y/o residuos, ya que ellos deben cumplir con los supuestos. En caso contrario el modelo de medias no es válido.
La figura 4 muestra que los residuos de la oxima A presentan un comportamiento normal.

En el histograma se observa una asimetría negativa de los datos respecto a la media. Además se observa la independencia de los residuos versus los valores ajustados. Finalmente en la imagen de orden de datos versus los residuos no se detectan patrones inusuales en los residuos.

La figura 5 muestra que los residuos de la oxima $\mathrm{B}$ presentan un comportamiento normal.

Además en el histograma se observa una asimetría positiva de los datos respecto a la media. En la gráfica de residuos se observa la independencia de los residuos versus los valores ajustados. Finalmente en el gráfico de orden de datos versus los residuos no se detectan patrones inusuales en los residuos.

La figura 6 muestra que los residuos de la oxima $\mathrm{C}$ presentan un comportamiento normal.

En el histograma se observa una asimetría negativa de los datos respecto a la media. En la gráfica de residuos se observa la independencia de los residuos versus los valores ajustados y finalmente en el gráfico de orden de datos versus los residuos no se detectan patrones inusuales en los residuos.

Como se aprecia en las figuras 4, 5 y 6 los residuos cumplen con los supuestos del modelo de medias, por lo tanto se puede afirmar que el modelo es válido.

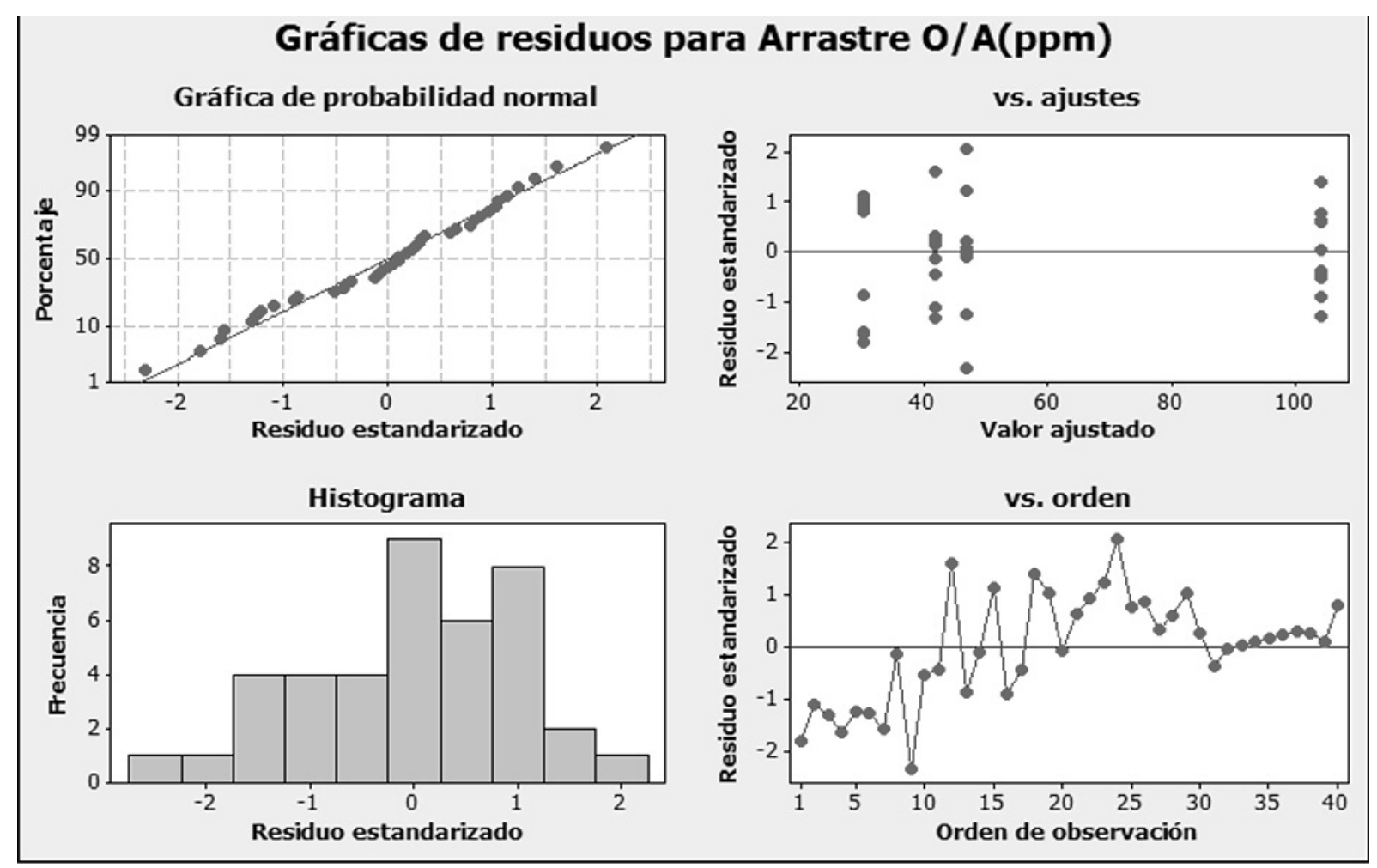

Figura 4. Gráfica de residuos de la oxima A.

Figure 4. Graphic of residues of oxime A. 


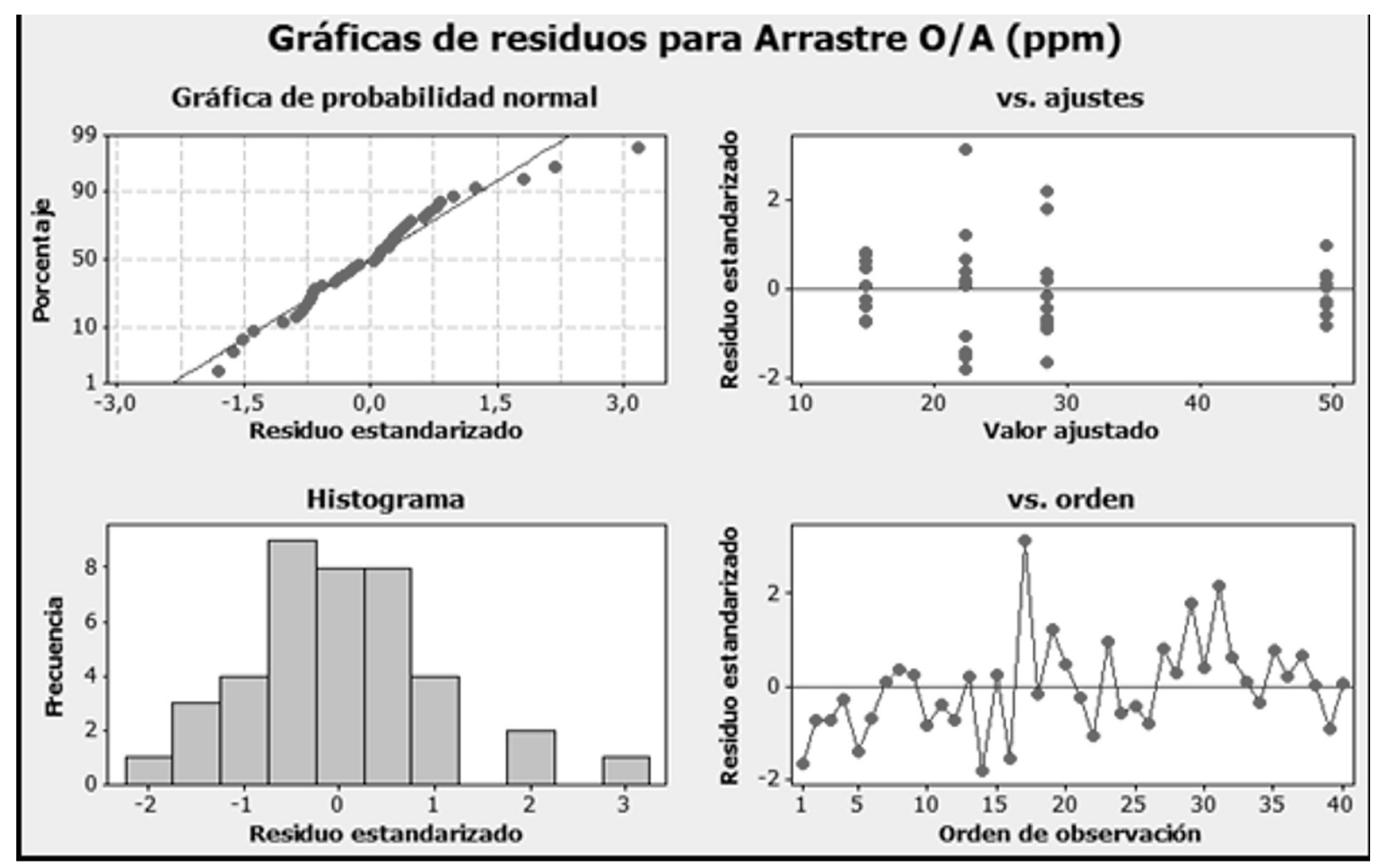

Figura 5. Gráfica de residuos de la oxima B.

Figure 5. Graphic of residues of oxime B.

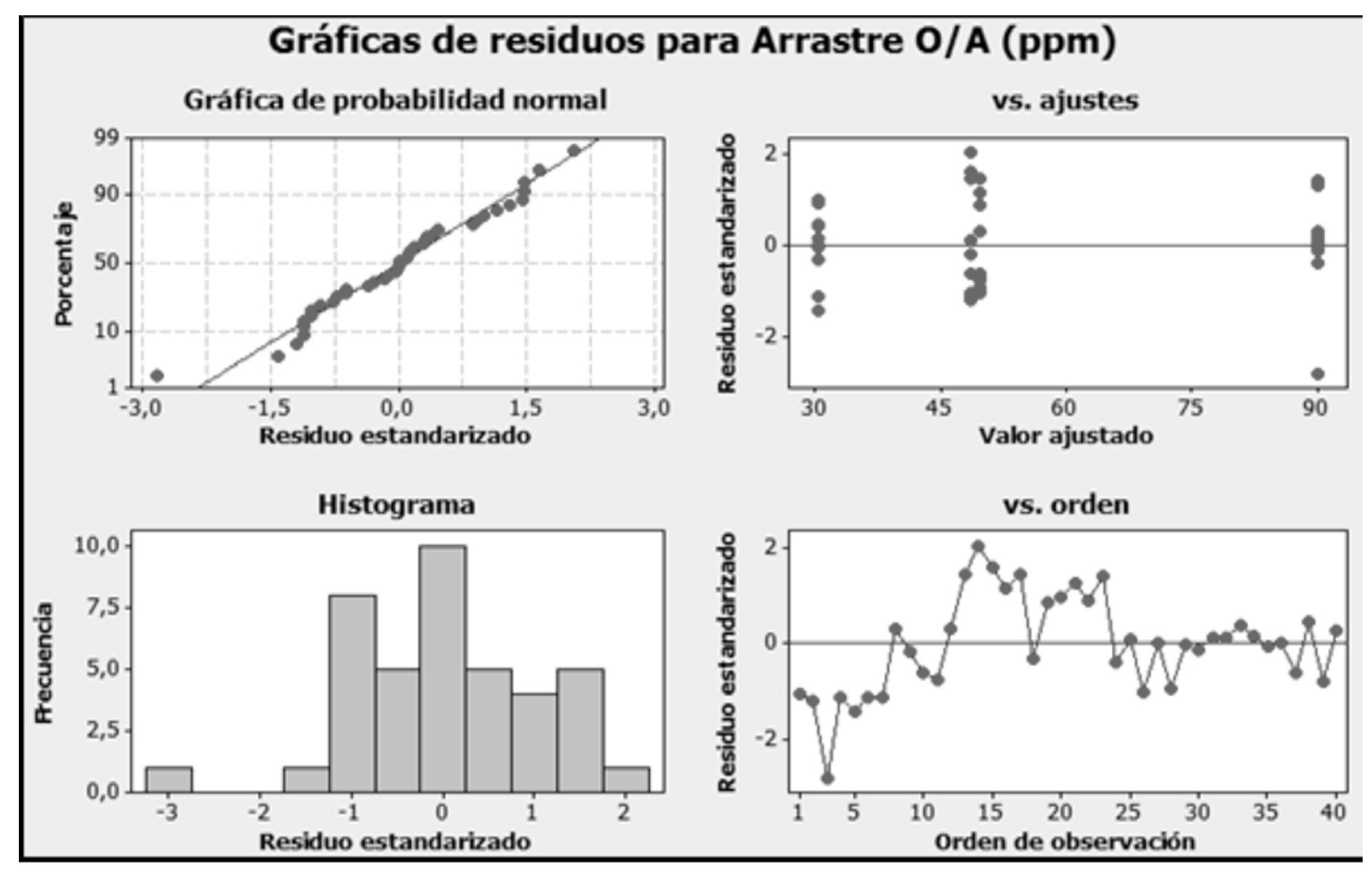

Figura 6. Gráfica de residuos de la oxima C.

Figure 6. Graphic of residues of oxime C. 


\section{CONCLUSIONES}

- El tipo de oxima es estadísticamente significativo en el arrastre O/A. Los niveles menores de arrastres se obtienen en la cetoxima, alcanzando un $40 \%$ y $20 \%$ inferior a las aldoximas y mezclas respectivamente.

- La ecuación del modelo de arrastre obtenida del análisis factorial para las distintas oximas está dado por: Arrastre $\mathrm{O} / \mathrm{A}=\mathrm{C}-\mathrm{B} *$ Temperatura $\mathrm{E} * \mathrm{pH}+\mathrm{F} *$ Agitación, donde $\mathrm{C}, \mathrm{B}, \mathrm{E}$ y $\mathrm{F}$ son constantes específicas de cada reactivo.

- La densidad, viscosidad y tensión interfacial disminuyen al incrementar la temperatura.

- Al aumentar la agitación en 200 rpm los arrastres O/A aumentan en promedio un $60 \%$ para el caso de las cetoximas, $55 \%$ en las mezclas y $68 \%$ en el caso de las aldoximas modificadas.

- $\mathrm{Al}$ aumentar la temperatura en $10^{\circ} \mathrm{C}$ los arrastres $\mathrm{O} / \mathrm{A}$ aumentan en promedio un $50 \%$ para el caso de las cetoximas, $40 \%$ en las mezclas y $55 \%$ en el caso de las aldoximas modificadas.

- Los residuos y/o errores cumplen con el supuesto del modelo, por lo que estadísticamente el modelo de medias es válido.

- La ecuación para cada reactivo es propia para las condiciones operacionales utilizadas, no es extrapolable a un sistema industrial.

\section{Agradecimientos}

Los autores agradecen a Basf por el financiamiento y soporte técnico durante la investigación. Además a la Universidad Católica del Norte y CICITEM por el apoyo recibido para el uso de equipamiento.

\section{REFERENCIAS}

[1] G. Vera, Hydroprocess, Vol.1, Santiago, Chile. 2010. Gecamin. pp 12-13.

[2] A. Patwardhan, T. Mundada, V. Morab y C. Srilatha, Chem. Eng. Sci. 65 (2010) 3.409-3.426.

[3] M.C. Ruiz, P. Lermanda y R. Padilla, Hydro. Eng. 63 (2001) 65-74.

[4] R. Semia y C. Nadiv, Chem. Eng. Sci. 34 (1995) 2.427-2.435.

[5] N. Denkov, N. Vankova, S. Tcholakova y T. Danner, Chem. Eng. Sci. 310 (2007) 570-589.

[6] M. Kraume, A. Paschedag, M. Weger y A. Gäbler, Chem. Eng. Sci. 61 (2006) 3.018-3.024.

[7] J. Lagissety, P. Das, R. Kumar y K. Gandhi. Chem. Eng. Sci. 41 (1986) 65-72.

[8] A. Sathyagal, D. Ramkrishna y G. Narsimhan. Chem. Eng. Sci. 51 (1996) 1.377-1.391.

[9] A. Kosshy, T. Das y R. Kumar, Chem. Eng. Sci. 43 (1988) 649-654.

[10] T. Tobin y D. Ramkrishna, AICHE J. 38 (1992) 1.199-1.205. 